\title{
Foreshock-like density cavity in the outflow region of magnetotail reconnection
}

\author{
C. L. Cai ${ }^{1,2,3}$, I. Dandouras ${ }^{1,2}$, H. Rème ${ }^{1,2}$, J. B. Cao $^{4,3}$, G. C. Zhou ${ }^{3}$, G. K. Parks ${ }^{5}$, and D. Fontaine ${ }^{6}$ \\ ${ }^{1}$ University of Toulouse, UPS, CESR, 9 avenue colonel Roche, 31028 Toulouse cedex 4, France \\ ${ }^{2}$ CNRS, UMR5187, 31028 Toulouse, France \\ ${ }^{3}$ State Key Laboratory of Space Weather, Center for Space Science and Applied Research, Beijing 100080, China \\ ${ }^{4}$ School of Astronautics, Beijing University of Aeronautics and Astronautics, Beijing 100191, China \\ ${ }^{5}$ Space Sciences Laboratory, University of California, Berkeley, CA 94720, USA \\ ${ }^{6}$ Centre d'Etudes des Environnements Terrestre et Planétaires, Centre National de la Recherche Scientifique, \\ 78140 Vélizy, France
}

Received: 20 May 2008 - Revised: 27 April 2009 - Accepted: 6 July 2009 - Published: 5 August 2009

\begin{abstract}
During Cluster spacecraft crossing of the magnetotail, a novel density depleted cavity in association with magnetic compressions in the outflow region of reconnection was observed. It contains intense reflected field-aligned particles, which are produced by a generation mechanism similar to that of the terrestrial foreshock, and hence manifests a foreshock-like morphology. In this cavity, reflected field-aligned proton beams were observed and simultaneously the feature of magnetic-mirror loss-cone proton distributions were found. Magnetic field fluctuations, especially quasi-monochromatic oscillations, were recorded. Both the leading egde and the ULF wave boundary of the ion foreshock are identified from the time sequence of proton and magnetic field observations. Just upstream of the leading egde of the ion foreshock, reflected field-aligned electrons were detected, whose distribution has a narrow bump-on-tail pattern. However, close to the shock front, reflected electrons with a broad bump-on-tail pattern was measured. These two different manifestations of reflected electrons reveal the differences in their microscopic physics of the reflecting process. Moreover, a part of incident ions was further accelerated in the cavity due to trans-time magnetic pumping which provides another possible mechanism in the multi-step acceleration processes in reconnection.
\end{abstract}

Keywords. Magnetospheric physics (Magnetotail) - Space plasma physics (Charged particle motion and acceleration; Magnetic reconnection)

\section{Correspondence to: C. L. Cai} (clcai@cssar.ac.cn)

\section{Introduction}

Magnetic reconnection plays a crucial role in the dynamics of the magnetotail. During magnetic reconnection, the lobe plasma is convected toward the central plasma sheet, and is driven out from the $\mathrm{X}$-type diffusion region in the form of ion jets in two opposite directions (Hones, 1979). The magnetic topological structures of these ion jets and their effects on the surrounding plasmas have been intensively investigated. For instance, reconnection generated flux ropes were observed (Hones, 1977; Slavin et al., 1989). Also, the interaction between the fast-moving flux rope and the lobe manifests as a bulge that compresses the lobe plasma, which is called traveling compressed plasma region (Slavin et al., 1984, 2005). Moreover, the ion outflows observed in the Earth's magnetotail reconnection show a wide variety of singular boundary layers, in addition to the well-known slow-mode shock boundary (Eriksson et al., 2004). On the basis of Geotail spacecraft data, it had been found that during magnetic reconnection a contact discontinuity exists binding two different plasma regions in the sheet, i.e., separating the shockheated plasmas from the Joule heated plasmas by magnetic diffusion (Hoshino et al., 2000). Computer simulations also show that a tangential discontinuity inside the plasmoid can form to separate the accelerated plasmas from the original plasma (Abe and Hoshino, 2001). Moreover, simulation results indicate the interaction between the fast reconnection jets and the original sheet plasmas associated with a magnetic loop can form a fast shock at the edge of the reconnection jet (Ugai, 1996, 1999).

In the Geospace, the most significant singular boundary created by high-speed plasmas confronting an obstacle is the

Published by Copernicus Publications on behalf of the European Geosciences Union. 
terrestrial magnetospheric bow shock. When the supersonic solar wind plasma carrying the interplanetary magnetic field encounters Earth's dipole magnetic field, the bow shock is formed, which slows down the solar wind plasma from supersonic to subsonic speeds and thermalises it. Simultaneously, a foreshock forms if a part of incident particles is reflected. The foreshock is a region upstream of the bow shock, which is magnetically connected to the bow shock and comprises both incident solar wind plasma and reflected particles. For the quasi-perpendicular shock, the foreshock is confined to the shock foot, while upstream of the quasiparallel shock, it occupies a much larger area (Balogh et al., 2005). The foreshock possesses complex kinetic processes, which give rise to accordingly unusual spatial structure (Bale et al., 2005; Eastwood et al., 2005). It is generally thought that a certain portion of the incident particles can be specularly reflected at the shock magnetic ramp (Paschmann et al., 1980; Gosling et al., 1982, Schwartz et al., 1983). Those reflected particles that have high enough velocity parallel to the magnetic field (hence their guiding center velocity along the shock normal is larger than the convection speed) will escape upstream along the field line to create a backstreaming field-aligned beam, which move both along the magnetic field line and simultaneously drift in the convective electric fields of the incident particles. Eventually, these backstreaming field-aligned beams can be found behind the tangential magnetic field line. Therefore, the upstream boundary of the foreshock is the locus of the tangential field lines, and the downstream boundary of the foreshock is the quasi-parallel part of the shock. The higher energy particles can be observed close to the upstream foreshock boundary, while the lower energy particles can be recorded further downstream. Just behind the tangent field line is the electron foreshock. The ion foreshock will be encountered downstream the electron foreshock. The reflected field-aligned ion beams with the smallest drift distance make up the upstream boundary of the ion foreshock, which is also called the leading ion foreshock boundary. Behind the leading ion foreshock boundary, field-aligned backstreaming ion distributions are observed. Deeper in the foreshock, diffuse backstreaming ion distributions are usually recorded (Meziane et al., 2001, 2004). Moreover, there exists a second boundary within the ion foreshock, closer to the quasi-parallel shock, usually referred to as the ULF foreshock boundary or the ion foreshock wave boundary (Le and Russell, 1992; Meziane et al., 1998). This boundary borders the domain of ULF wave activity.

In this paper, the following particular scenario is considered, where the reconnection ion jets, especially those traveling earthward, collide with the original plasmas at rest before reconnection. The reconnected magnetic field lines carried outward by the high-speed ion jet emanating from the X-type diffusion region can be blocked by the original magnetic field lines bordering the outflow region. Therefore, in the edge of outflow region, there exists a region where field lines are piled up. When the magnetic compression, or in other words the impact of ion jet, is intense enough to cause some kinds of singular boundary layer such as a discontinuity or a shock to form, it isn't hard to find that there is a remarkable similarity in the underlying physical process between the present situation and that of the bow shock. However, since there are many differences in the respective environments of two cases, whether the ion jets can interact with the original plasmas in a bow shock-like manner is still unclear. Also, since the existence of reflected particles is an intrinsic feature of collisionless shock, can some particular characteristics relevant to foreshock-like structure be exhibited in the vicinity of a steepened boundary of the field lines piled up region in the edge of outflow region?

Here, we present some reliable observations of a singular boundary of an ion jet that is formed due to its interaction with the original plasmas, and report observations of a density depletion cavity with a foreshock-like structure in the outflow region of magnetotail reconnection. First, an overview of the reconnection layer encountered by the spacecraft is given. Then, particle kinetics and magnetic field measurements are analyzed in an attempt to reveal the remarkable characteristic structures in the density cavity that manifests a highly similar morphology to the foreshock region.

\section{Observations}

\subsection{Overview}

During the time interval 00:30-00:50 UT on 6 August 2003, the 4 Cluster spacecraft crossed the near-Earth magnetotail at $X_{\mathrm{GSM}}=-17 R_{E}$. Figure 1 shows the magnetic field measurements from the FGM experiment (Balogh et al., 2001) and proton measurements from the CIS-CODIF instrument (Rème et al., 2001). Cluster spacecraft C4 data are shown, but because of the very small size of the 4 spacecraft tetrahedron $(\sim 200 \mathrm{~km})$ all spacecraft measured very similar observational features. While the spacecraft was in the plasma sheet of the Southern Hemisphere, due to a negative $B_{X}$, it suddenly observed an earthward fast proton flow with a total velocity up to $\sim 610 \mathrm{~km} / \mathrm{s}$ in the interval 00:33:20 00:35:10 UT. Accompanied with this plasma ejection is a strong magnetic field variation. The magnetic field magnitude of the leading boundary increases from $-20 \mathrm{nT}$ to $-34 \mathrm{nT}$ in $B_{X}$ and from 3 to $10 \mathrm{nT}$ in $B_{Z}$. In this jet, the proton density decreases from $0.72 \mathrm{~cm}^{-3}$ to $0.32 \mathrm{~cm}^{-3}$, about half of the original level. The oxygen ion abundance is very low and not shown here.

After passing the density cavity, the spacecraft subsequently observed two density dips at 00:37:30 UT and 00:43:40 UT, respectively. Finally, the spacecraft met a tailward fast flow with a total velocity up to $\sim 600 \mathrm{~km} / \mathrm{s}$ in the interval 00:45-00:49 UT. In this event, $B_{0}=25 \mathrm{nT}, T_{H}=5 \mathrm{keV}$, and $n_{H}=0.7 \mathrm{~cm}^{-3}$, where $B_{0}$ is the magnetic field in unperturbed plasma sheet, $T_{H}$ and $n_{H}$ are the proton temperature 


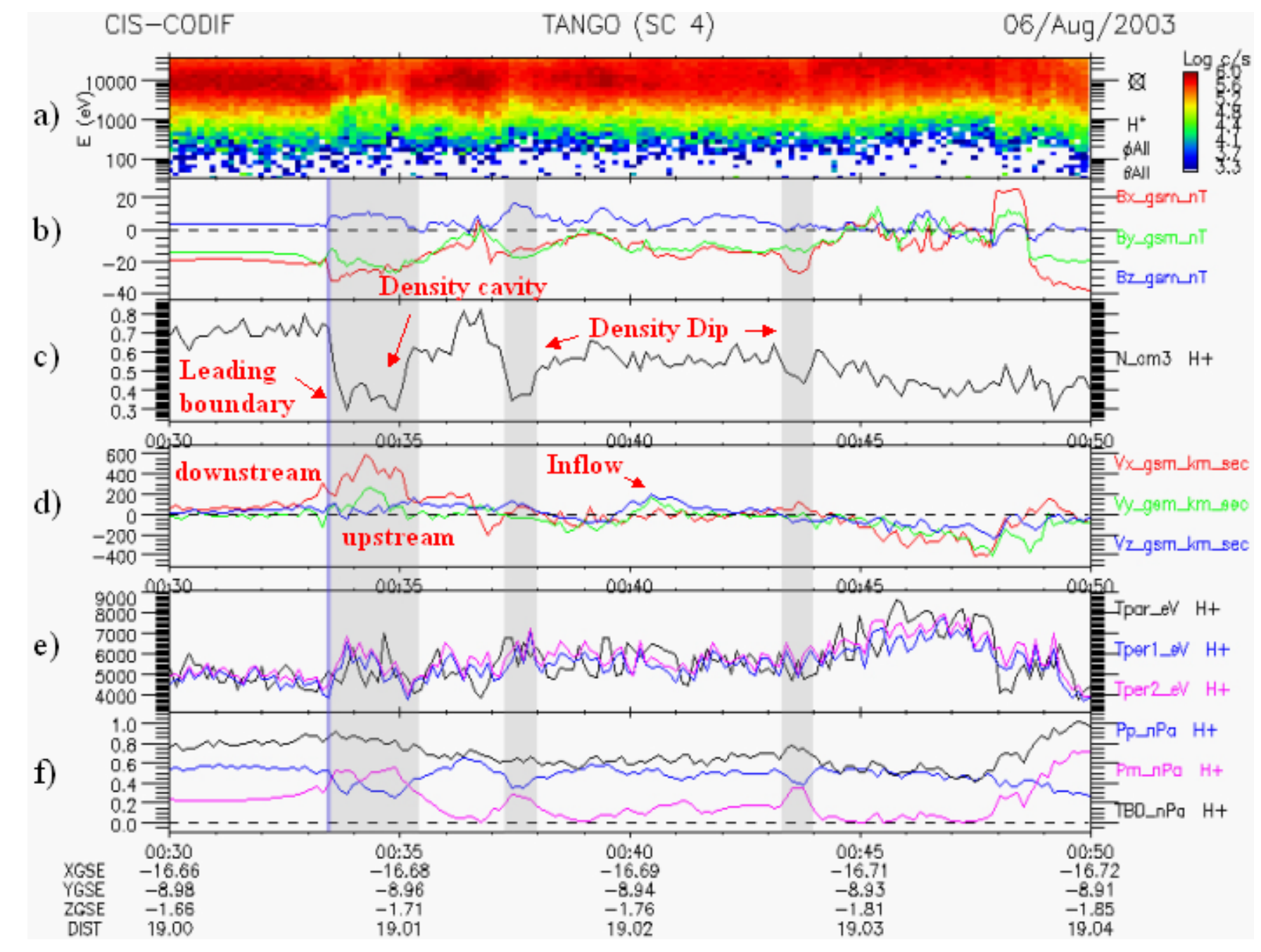

Fig. 1. Magnetic field and proton measurements from C4. From top: (a) time-energy spectrogram, (b) components of magnetic field, (c) and (d) proton density and bulk velocities respectively, (e) proton pressure components parallel and perpendicular to the magnetic field, and (f) magnetic pressure, proton pressure and total pressure respectively. The shadows denote the density cavity and two density dips, respectively.

and density, respectively. The Alfvén speed $V_{A}$, proton Larmor radius $\rho_{H}$, and proton inertial length $c / \omega_{\rho H}$ are respectively $V_{A}=650 \mathrm{~km} / \mathrm{s}, \rho_{H}=410 \mathrm{~km}$ and $c / \omega_{p H}=270 \mathrm{~km}$.

\subsection{The reconnection layer structruce and the steep- ened leading boundary of the earthward jet}

Flow reversal in the magnetotail is usually interpreted as a reconnection event. In the present case, the encountered reconnection layer is schematically shown in Fig. 2. The nascent magnetic flux ropes can be carried out by the ion jet emanating from the diffusion region (Slavin et al., 2005). They are blocked by the original magnetic field lines bordering the outflow region and hence are piled up at the edge of outflow region. When the magnetic compression is intense enough, it is believed that plasma depletion phenomena usually take place due to pressure balance, and in most cases in association with some kind of singular boundaries. Here the earthward jet in the form of a density cavity with a sharp leading boundary is interpreted as exactly that case. In addition, this sharp leading boundary can be considered in principle as an MHD discontinuity or shock and hereinafter we refer to it as a shock. Here, the term "shock" is used in a rather general meaning, that is, if the front becomes steep enough that the entropy increases across it and the changes become irreversible, we refer to the front as a "shock" (Southwood and Kivelson, 1992).

By the magnetic field timing analysis, the normal direction and speed of the shock are estimated to be $\mathbf{n}_{\mathrm{GSM}}=(0.45$, $-0.01,0.89)$ and $78 \mathrm{~km} / \mathrm{s}$, respectively. The duration of its crossing is $14 \mathrm{~s}$. Hence its thickness is estimated to be $1100 \mathrm{~km}$, four times the proton inertial length. In the GSM 


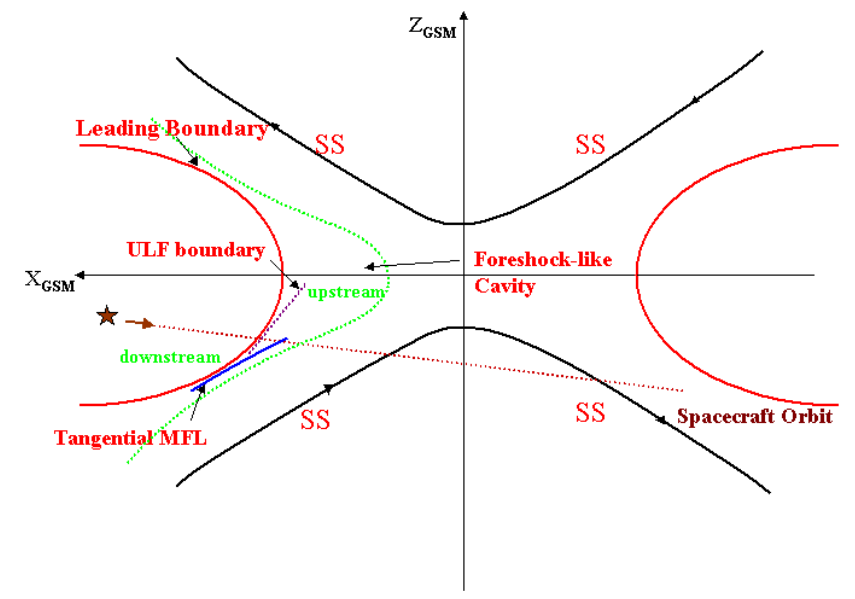

Fig. 2. A schematic of the reconnection layer encountered by the spacecraft and the boundaries in the cavity. The two black curves represent the pair of slow-mode separatrices. The dashed green line depicts the local magnetic configuration of the field line piled up region. The red curve in the left-side describes the shock front, i.e., the sharp leading edge of the ion jet. The blue line signifies the magnetic field line tangent to the quasi-perpendicular shock front. The star and the dashed brown line denote the spacecraft and its orbit. More details can be founded in the text.

frame, the magnetic fields upstream and downstream of the shock are $\mathbf{B}_{u}=(-29,-23,7)$ and $\mathbf{B}_{d}=(-21,-16,3)$, respectively. Therefore the angle between the magnetic field and the normal to the shock is about $80^{\circ}$, which means that the front encountered is a nearly proper perpendicular part of the shock. Moreover, we have approximately $\Delta \boldsymbol{B} \cdot \boldsymbol{n}=0$ and $\left(\mathbf{B}_{u} \times \mathbf{B}_{d}\right) . \mathbf{n}=0$, where $\Delta \mathbf{B}$ is $\mathbf{B}_{u}$ minus $\mathbf{B}_{d}$, and hence verify the magnetic coplanarity. In addition, in the shock rest frame, the bulk velocities upstream and downstream of the shock are $\mathbf{V}_{u}=(550,242,-74)$ and $\mathbf{V}_{d}=(270,-130,-54)$, respectively. Thus the calculated upstream and downstream normal velocities are $179 \mathrm{~km} / \mathrm{s}$ and $75 \mathrm{~km} / \mathrm{s}$, respectively. The ratio of the upstream normal velocities to the downstream normal velocity is about 2.4 , while the upstream and downstream densities are $0.72 \mathrm{~cm}^{-3}$ and $0.33 \mathrm{~cm}^{-3}$, respectively. The ratio of the downstream density to the upstream density is about 2.2. Hence mass conversation is approximately satisfied. The estimation on the full set of jump conditions is quantitatively less consistent. The reason is due to the non-stationarity behavior of this cavity because of the explosive reconnection jets. Taking account of this fact, the upstream parameters may be highly time-dependent. In addition, the calculated velocity of the ion jet includes the contribution from reflected backstreaming particles and should be less than the actual incident velocity. Therefore, although the calculated upstream normal velocity $(\sim 179 \mathrm{~km} / \mathrm{s})$ is even smaller than the slow-mode speed, which is estimated to be $\sim 250 \mathrm{~km} / \mathrm{s}$, it seems reasonable that this leading boundary is identified as a time-dependent slow-mode transition layer.
It is worth noting that this leading boundary inside the outflow region that separates the accelerated plasmas from the original plasma should not be confused with the well-known Petschek-type slow-mode shock around the reconnection region since it moved earthward. Otherwise, it would not be the leading boundary of the jet and the bulk flow would be encountered first.

Density dips encountered in reconnection layers are usually identified as Petschek-type slow-mode separatrices (Cattell et al., 2005; Borg et al., 2005). Here the two density dips encountered at 00:37:30 UT and 00:43:40 UT are regarded as the slow-mode separatrices bounding the earthward side and tailward side outflow regions respectively. Between the slowmode separatrices, there is the southern inflow region. Thus nearly in the middle of this region at 00:40:30 UT, a peak of $V_{z}$ was recorded as shown in the fourth panel in Fig. 1, which is identified as the inflow velocity with speed up to $\sim 200 \mathrm{~km} / \mathrm{s}$.

It is worthy to note that in the intervals $00: 36: 40-00: 37$ UT and 00:47:50-00:48:30 UT, the spacecraft encountered the neutral line and the outer current sheet respectively due to the very rapid large-scale shift of the current sheet, which are phenomena frequently occurring in the thin current sheet and usually referred to as the flapping motion (Sergeev et al., 2003; Cai et al., 2008). The fifth panel in Fig. 1 shows the components of the proton temperature. It can be seen that in both the earthward and tailward ion jets, the proton temperature rose, apparently due to the heating by both the magnetic diffusion and by the slow-mode separatrices.

\subsection{Incident and backstreaming protons inside the cavity}

The global morphology of the density cavity can be established from the observational characteristics of particle kinetics. Figure 3 gives the spectrogram of the pitch angle and azimuthal angle of protons recorded by $\mathrm{C} 4$ in 2 energy ranges. In the cavity, from 00:34:05 to 00:34:44 UT as shown in the sixth panel, intense incident protons within the energy range $1.5-40 \mathrm{keV}$ in the anti-parallel magnetic field direction were observed. Simultaneously, a very collimated backstreaming beam distribution in the parallel magnetic field direction was recorded. The fourth and fifth panels show the proton behaviors in the energy range $50-1500 \mathrm{eV}$. It can be seen that in the cavity the pitch angles of these protons with lower energies display a narrow profile between $50^{\circ}$ and $70^{\circ}$. Also, along the negative Y-direction they are within an azimuthal angle profile centered between $-80^{\circ}$ and $-100^{\circ}$.

Figure 4 shows the proton distribution functions for all the twelve data acquisition periods in the cavity (Each ion data acquisition period is here two spacecraft spins, i.e. $8 \mathrm{~s}$ ). It isn't difficult to find that from the third period to the last one, all these distribution functions show an apparent loss-cone distribution in the direction parallel to the magnetic field. 


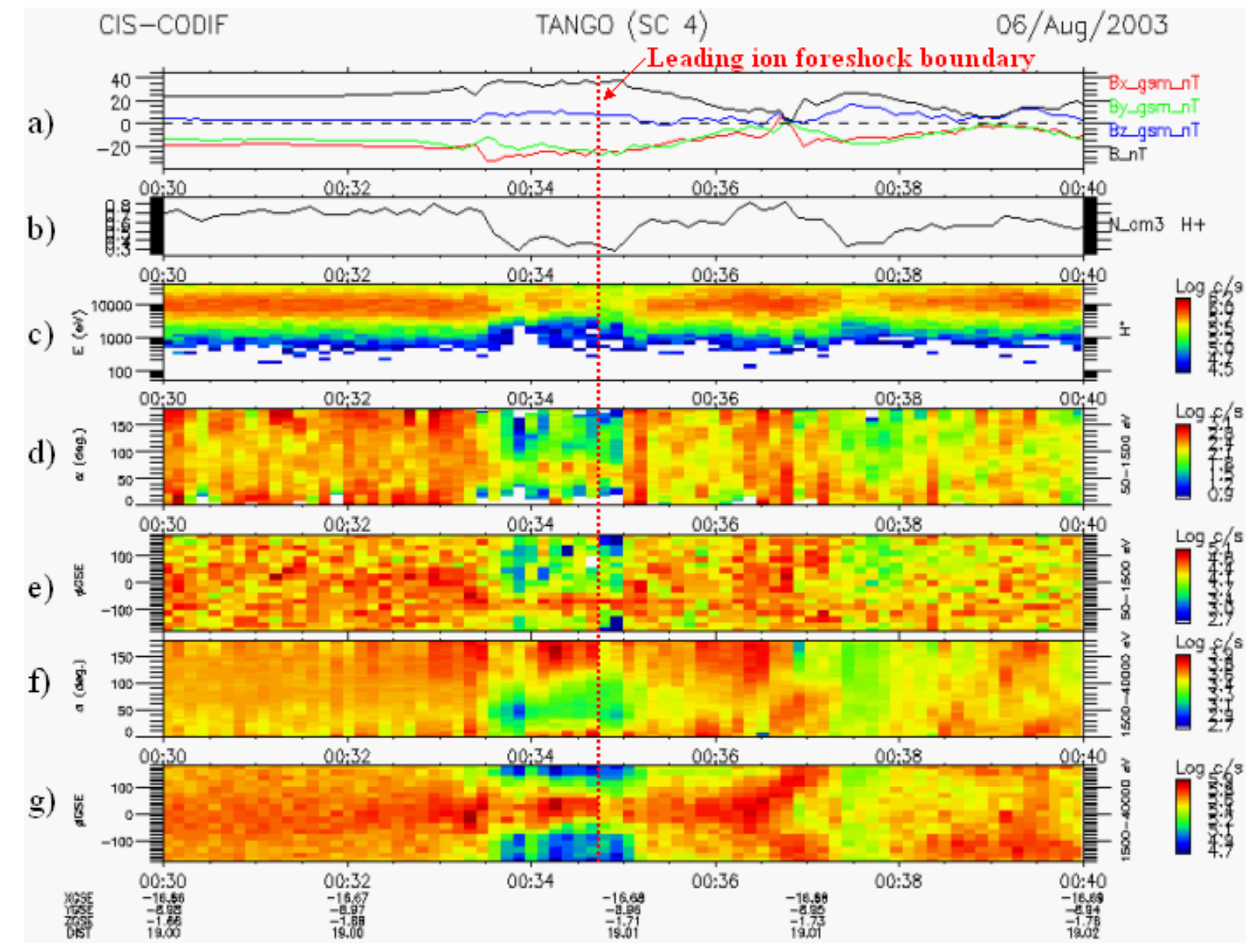

Fig. 3. A snapshot of the proton measurements in the cavity. The third panel is the time-energy spectrogram. The fourth and fifth panels show the spectrogram of the pitch angle and azimuthal angle respectively for protons within the energy range $50-1500 \mathrm{eV}$, and the sixth and seventh panels show those for protons within the higher energy range $1.5-40 \mathrm{keV}$.

Figure 5 shows an extract of these distribution functions in the seventh period at 00:34:13 UT.

As mentioned above, the depleted density cavity, in association with a steepened leading boundary, is generated by the piled up magnetic field lines, which are carried out by the ions jet emanating from the diffusion region. The present scenario resembles highly the case that when the solar wind impacts the magnetosphere, a curved bow shock front forms. In contract to the hydrodynamics shock, in which no information can be transmitted to upstream region, the existence of reflected particles back to the upstream region is an intrinsic feature of collisionless MHD shock. Here the observed collimated backstreaming beam is interpreted to be those reflected particles by the steepened leading boundary, that is, whose generation mechanism is exactly similar to that of the foreshock (Paschmann et al., 1980; Gosling et al., 1982; Schwartz et al., 1983).
In Fig. 2, a schematic is shown in which the blue line indicates the magnetic field line tangent to the perpendicular part of the curved shock front. The specularly reflected particles with high field-aligned velocity move both along the magnetic field line and simultaneously drift in the convective electric fields of the incident protons. Eventually, these backstreaming field-aligned beams can be found behind the tangential magnetic field line, along which they escape upstream from the shock front. In the present case, the leading ion foreshock boundary is encountered at 00:34:44 UT while the spacecraft crossed the foreshock region from downstream to upstream, thus no field-aligned ion beam was observed after this time.

One issue of the interpretation of the reconnection outflow region as analogous to the bow shock-foreshock region must be clarified here. For the bow shock, it is a fast-mode type, while in the present case the sharp boundary is a slow-mode 

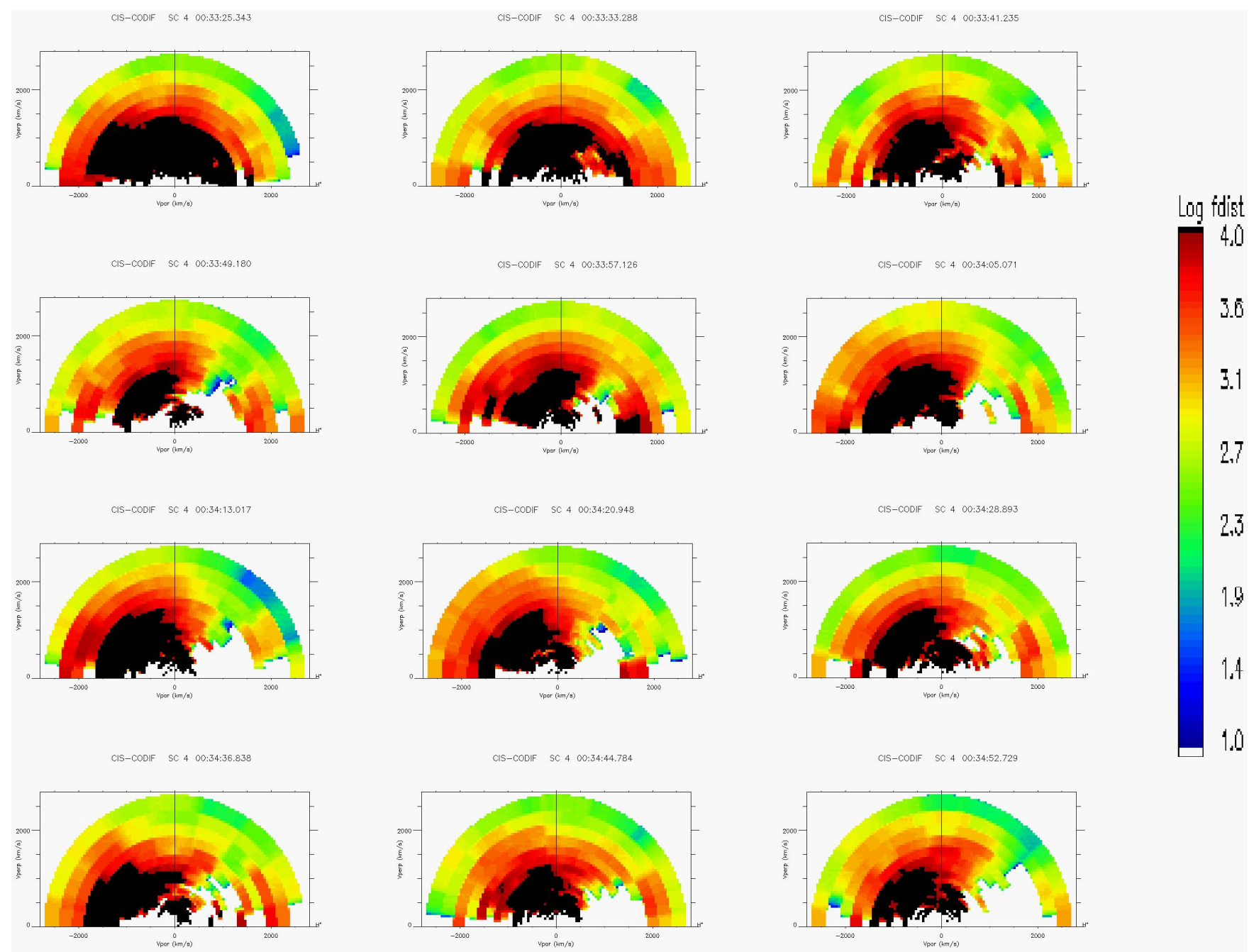

Fig. 4. Proton distribution functions for all the twelve data acquisition periods in the cavity.

shock based on the above investigation. However, whatever the shock is, the crucial point is that in both cases a steepened current sheet is generated and can act as a reflecting layer. The interpretation of the generation mechanism of those field-aligned backstreaming particles in the cavity is a natural explanation.

Apart from the specularly reflected particles, there are also other populations flowing back upstream of the shock by magnetic mirroring or in any other way. For example, several populations can be identified in Fig. 5. The loss-cone is the most prominent characteristic in this distribution due to magnetic mirroring. The population which fills the edge of the loss-cone with a large parallel velocity is the specularly reflected field-aligned beam. Moreover, there are two populations of incident protons: the major population has lower velocity, the minor population has higher velocity. Compared with the distributions in the other periods, we can infer that the population with lower velocity is the normal incident par- ticles, while the population with higher velocity is apparently accelerated. It demonstrates the possibility that the incident particles can be further energized in the field line piled up region in the outflow region of reconnection, especially in the case that the singular boundary condition occurs. The energisation mechanism here is supposed to be a trans-time magnetic pumping (Chen, 1984). Therefore, it provides another possible acceleration mechanism for the particles in their multi-step acceleration processes in the reconnection (Imada et al., 2007).

\subsection{ULF waves in the cavity}

The presence of intense low frequency electromagnetic fluctuations is an inherent feature of the ion foreshock region (Eastwood et al., 2002, 2004). ULF waves in association with backstreaming diffuse ion distributions are usually observed deep into the ion foreshock region, approaching to the quasi-parallel shock front (Bame et al., 1980; Meziane et al., 
2001, 2004; Cao et al., 2009; Fu et al., 2009). Those ULF waves are believed generated by the backstreaming fieldaligned beams, and the beams then become diffused. The upstream boundary of the ULF wave activity region is commonly called the ULF foreshock boundary or the ion foreshock wave boundary. Thus this boundary is between the backstreaming field-aligned beam distributions and diffuse distributions in the ion foreshock.

Figure 6 shows a snapshot of the magnetic field measurements with $1 \mathrm{~s}$ time resolution and the proton density in the cavity. The red, gray and blue shadows denote respectively the time intervals of the shock ramp, ULF waves and the backstreaming field-aligned beam. Both the leading egde and the ULF wave boundary of the ion foreshock are identified from the time sequence of proton and magnetic field observations. It is important to note that in the interval of the shock ramp, a bipolar structure of $B_{Y}$ was observed. It is inferred to be excited by the surface current in the shock front that results from the decoupling of the ion motion with the electron motion due to the finite Larmor radius effect (Bale et al., 2005). Shortly after the shock ramp, ULF wave activity was observed in the interval 00:33:40-00:34 UT. Especially, in the second panel, a quasi-monochromatic $B_{X}$ oscillation was found. Its period is about $9 \mathrm{~s}$, while the period of the fast magnetosonic waves upstream the bow shock is typically about 30 s (Eastwood et al., 2002, 2004). Also, just after the ULF wave region, the ion foreshock was encountered. It can be seen that the wave observations are well coincident with the particle kinetic measurements. The field-aligned beam distributions were not observed in conjunction with ULF waves, and the time gap between the ULF boundary and the backstreaming field-aligned beam distribution is less than one ion data acquisition period. Of course, in addition to the quasi-monochromatic ULF waves, intense higher frequency magnetic field turbulences can be found in the entire foreshock region including downstream of the shock front.

\subsection{Backstreaming electrons in the cavity}

Figure 7 shows 3 selected electron distributions parallel, antiparallel and perpendicular to the magnetic field from the PEACE experiment (Johnstone et al., 1997). The first and second distributions parallel to the magnetic field show a global enhancement, which represents a broad type of bumpon-tail pattern, while the third electron distribution parallel to the magnetic field has a narrower bump compared with that of the first and second distributions, which we refer to as a narrow type of bump-on-tail pattern (Fitzenreiter et al., 1984). The distributions recorded in other periods in the cavity have not obvious variation and are thus not shown here.

The two type bump-on-tail distributions are interpreted as the reflected electrons by the specular reflecting mechanism. However, the difference in their manifestations reveals the difference in their microscopic physics of reflecting process. The narrow bump is caused by the electrons suffering a re-

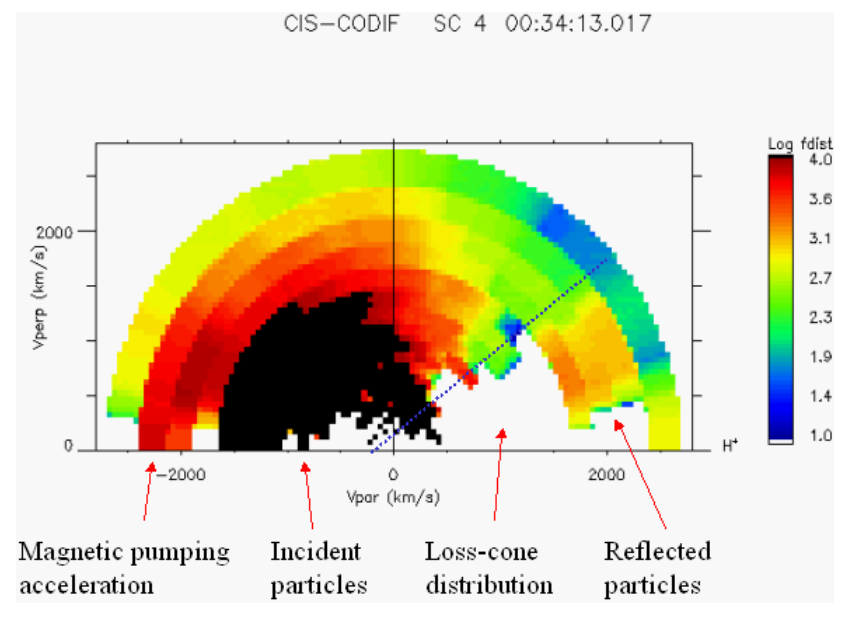

Fig. 5. Proton distribution function in the sixth data acquisition period in the cavity at 00:34:05 UT. Several populations with different kinetic characteristics are identified. The feature of loss-cone distribution is lined out.

flecting process that has a very short interaction time with the front, that is, interact only with the shock precursor and cannot reach the ramp itself. Furthermore, there is a second class of backstreaming electrons, which succeed to penetrate deeper the front and interact with the ramp (Lembège and Savoini, 2002). In this case, the trapped electrons can gain sufficient energy, including the parallel energy, from the shock electrostatic field while they excurse along the shock front. Corresponding to the two types of electron reflecting mechanisms, as revealed in simulations, there are two types of electron distributions parallel to the magnetic field, the narrow type of the bump-on-tail pattern and the broad type (Savoini and Lembège, 2001). The narrow type corresponds to the reflecting population that has lower parallel velocity, while the broad one corresponds to electrons that spend some time interacting with the macroscopic fields at the shock front and thus have higher parallel velocity. What kind of distribution can be found in the foreshock depends on the relative location of the shock geometry, and sometimes both types can coexist at the same location.

The narrow type of bump-on-tail pattern represents the population in the electron foreshock in a conventional meaning, that is, similar to the backstreaming field-aligned ions, backstreaming field-aligned electrons will also move along the magnetic field line and simultaneously drift in the convective electric fields, but within a smaller distance. It is coincident with the fact that these reflected electrons were observed at 00:34:54 UT, shortly after the passing of the leading ion foreshock boundary when the spacecraft crossed the foreshock region from downstream to upstream. However, the broad type of bump-on-tail pattern was observed within the two consecutive spin periods at 00:33:31 UT and 00:33:35 UT, respectively, very close to the shock front. They are the second class of backstreaming 


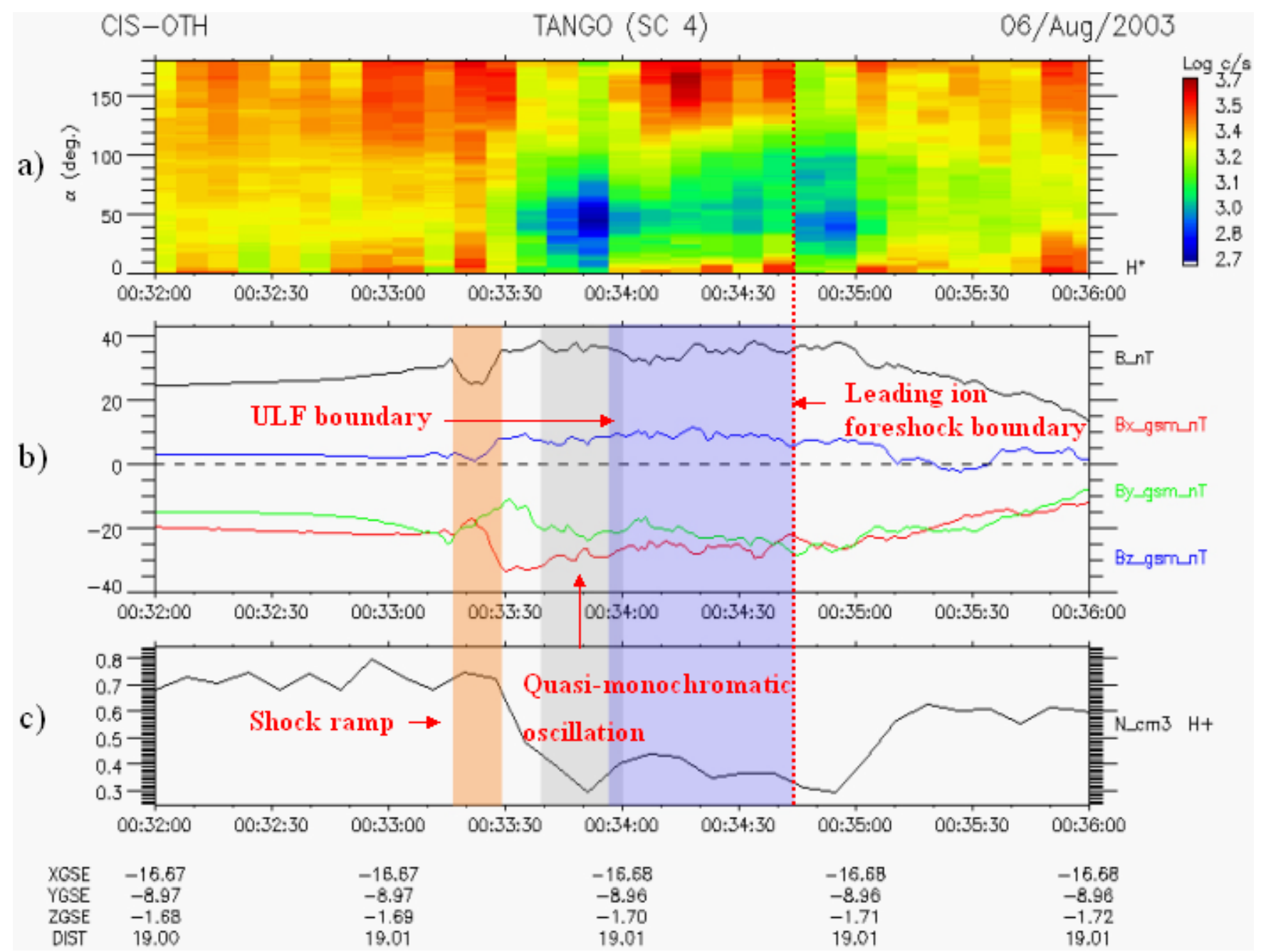

Fig. 6. A snapshot of the magnetic field measurements with 1s resolution and the proton density in the cavity. The red, gray and blue shadows denote respectively the time intervals of the shock ramp, ULF waves, and the backstreaming field-aligned beam.

electrons mentioned above, that is, electrons that interact with the ramp for a much longer time and gain more energy. This measurement gives evidence on the difference in their microscopic physics of reflecting process. It also indicates the remarkable impact of the local shock geometry on the electron kinetics. For the proper perpendicular part of the shock with a larger angle between the shock normal and the magnetic field direction, the electrons suffering a reflecting process that has a very short interaction time with the front. In contrast, for the quasi-perpendicular part with a smaller angle, the shock electrostatic field will make its contribution to the electron dynamics via local trapping and acceleration (Lembège et al., 2004).

\section{Discussion and conclusion}

We first give a summary on the morphology of the density cavity, which is analogous to the foreshock region. During the interval 00:33:16-00:33:30 UT, the spacecraft crossed the shock front from the downstream to the upstream. Just close to the front, in two consecutive spin periods at 00:33:31 UT and 00:33:35 UT, respectively, reflected electron distribution with a broad type of bump-on-tail pattern was observed. Then in the interval 00:33:40-00:34:00 UT, ULF waves, especially a quasi-monochromatic $B_{X}$ oscillation, were recorded. Just passing the ULF boundary, in the interval 00:34:05-00:34:44 UT, specularly reflected field-aligned proton beams were measured. Shortly after the passing of the leading ion foreshock boundary, corresponding to the spin period at 00:34:54 UT, specularly reflected field-aligned electrons, whose distribution has a narrow type of bump-ontail pattern, were detected. Moreover, magnetic-mirror losscone proton distributions in the cavity were observed.

Since the spacecraft had crossed both the left-side and the right-side outflow regions, a question arises as to why it didn't observe the same thing on the right-side. There are two possible reasons. The first is that in comparison to the 


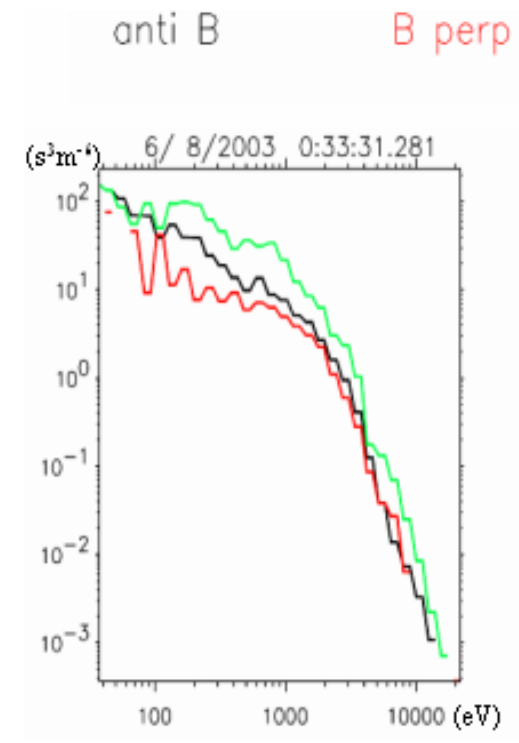

a)

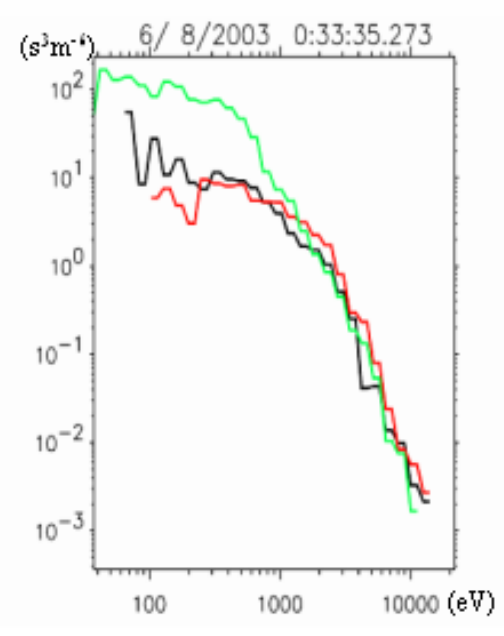

b)

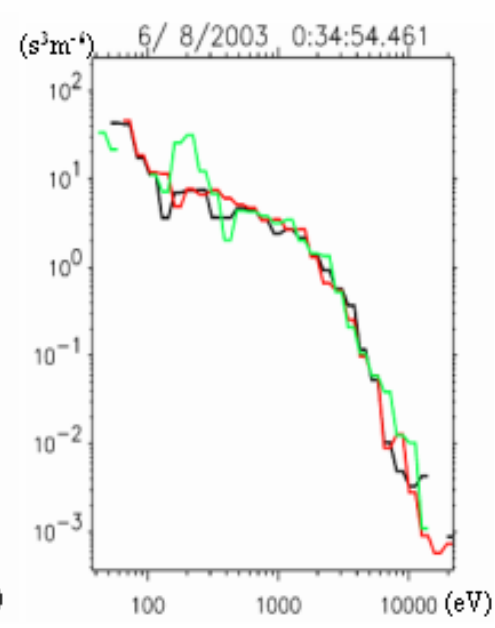

c)

Fig. 7. Selected electron distributions parallel, anti-parallel, and perpendicular to the magnetic field in the cavity. The first and second distributions parallel to the magnetic field manifest the broad type of bump-on-tail pattern, while the third distribution shows the narrow type of bump-on-tail pattern.

situation of flowing earthward, it is easier for the ion jet to push away its barrier while flowing tailward. The second is possibly due to the particular interaction manner of the reconnection jets with the rest plasma, that is, the jets are explosive and are bounded by a narrow slow-mode shock pair as mentioned above. Hence the shock and its accessories, if they can be generated at the edge of jets, will be localized both in space and time.

In addition, it is interesting to interpret the foreshocklike cavity in the viewpoint of a coherent structure. In the previous investigations, some transient structures such as hot diamagnetic cavities, foreshock cavities and short largeamplitude magnetic structures, have been reported in the upstream regions of the Earth's bow shock. Recently, the Cluster and Double Star satellites observed intense plasma density holes, with a characteristic dimension of ion gyroradius, upstream of the bow shock (Parks et al., 2006). Likewise, much attention has been paid on the coherent structures in the reconnection region, especially those electrostatic coherent structures in the diffusion region.

Large-amplitude solitary waves, identified as electron holes, have been observed during the passage of a magnetotail reconnection neutral line. These electron holes were generated near the outer edge of the plasma sheet, within and at the edge of a density cavity, at distances on the order of a few ion inertial lengths from the center of the current sheet (Cattell et al., 2005). These density cavities accompanied with electron holes can also be found in computer simulations (Drake et al., 2003). Therefore, large-scale coherent structures such as density cavity, which usually have a character- istic scale on the ion inertial length, seem to be an organic part of the reconnection and closely relevant to the smallscale electrostatic coherent structures. Coherent structures are usually responsible for those nonlinear microphysics in space plasma environment. For example, the electron holes are believed to participate in the electron energisation process (Drake et al., 2005). The present analysis shows that the density cavity can also act as the accelerator for ions to provide further acceleration. Our investigation here gives an insight into the significant role played by the self-organized larger-scale coherent structures in the collisionless magnetic reconnection region.

Acknowledgements. This work is supported by the National Natural Science Foundation of China (NSFC) under Grant No. 40621003, 40523006, 40574067 and the National Basic Research Program of China 2006CB806305 and the Specialized Research Fund for State Key Laboratories and the French National Space Agency (CNES). C.L.C. acknowledges the French Embassy in China for his government scholarship.

Topical Editor I. A. Daglis thanks two anonymous referees for their help in evaluating this paper.

\section{References}

Abe, S. and Hoshino, M.: Nonlinear evolution of plasmoid structure, Earth Planets Space, 53, 663-671, 2001.

Bale, S. D., Balikhin, M. A., Horbury, T. S., Krasnoselskikh, V. V., Kucharek, H., Möbius, E., Walker, S. N., Balogh, A., Burgess, D., Lembege, B., Lucek, E. A., Scholer, M., Schwartz, S. J., 
and Thomsen, M. F.: Quasi-perpendicular shock structure and processes, Space Sci. Rev., 118, 161-203, 2005.

Balogh, A., Carr, C. M., Acua, M. H., Dunlop, M. W., Beek, T. J., Brown, P., Fornacon, K.-H., Georgescu, E., Glassmeier, K.H., Harris, J., Musmann, G., Oddy, T., and Schwingenschuh, K.: The Cluster Magnetic Field Investigation: overview of in-flight performance and initial results, Ann. Geophys., 19, 1207-1217, 2001, http://www.ann-geophys.net/19/1207/2001/.

Balogh, A., Schwartz, S. J., Bale, S. D., Balikhin, M. A., Burgess, D., Horbury, T. S., Krasnoselskikh, V. V., Kucharek, H., Lembege, B., Lucek, E. A., Mobius, E., Scholer, M., Thomsen, M. F., and Walker, S. N.: Cluster at the Earth's Bow Shock: Introduction, Space Sci. Rev., 118, 155-160, 2005.

Bame, J. R., Moreno, G., Lazarus, A. J., and Sullivan, J. D.: Deceleration of the solar wind upstream from the Earth's bow shock and the origin of diffuse upstream ions, J. Geophys. Res., 85, 2981-2990, doi:10.1029/JA085iA06p02981, 1980.

Cai, C. L., Dandouras, I., Rème, H., Cao, J. B., Zhou, G. C., and Parks, G. K.: Cluster observations on the thin current sheet in the magnetotail, Ann. Geophys., 26, 929-940, 2008, http://www.ann-geophys.net/26/929/2008/.

Cao, J. B., Fu, H. S., Zhang, T. L., Reme, H., Dandouras, I., and Lucek, E.: Direct evidence of solar wind deceleration in the foreshock of the Earth, J. Geophys. Res., 114, A02207, doi:10.1029/2008JA013524, 2009.

Cattell, C., Dombeck, J., Wygant, J., Drake, J. F., Swisdak, M., Goldstein, M. L., Keith, W., Fazakerley, A., Andre, M., Lucek, E., and Balogh, A.: Cluster observations of electron holes in association with magnetotail reconnection and comparison to simulations, J. Geophys. Res., 110, A01211, doi:10.1029/2004JA010519, 2005.

Chen, F. F.: Introduction to Plasma Physics and Controlled Fusion, Vol 1: Plasma Physics, Second Edition, Plenum Press, 1984.

Drake, J., Swisdak, M., Cattell, C., Shay, M., Rogers, B., and Zeiler, A.: Formation of electron holes and particle energization during magnetic reconnection, Science, 299, 873-877, 2003.

Drake, J. F., Shay, M. A., Thongthai, W., and Swisdak, M.: Production of energetic electrons during magnetic reconnection, Phys. Rev. Lett., 94, 095001, doi:10.1103/PhysRevLett.94.095001, 2005.

Eastwood, J. P., Balogh, A., Dunlop, M. W., Horbury, T. S., and Dandouras, I.: Cluster observations of fast magnetosonic waves in the terrestrial foreshock, Geophys. Res. Lett., 29, 2046, doi:10.1029/2002GL015582, 2002.

Eastwood, J. P., Balogh, A., Mazelle, C., Dandouras, I., and Rème, H.: Oblique propagation of $30 \mathrm{~s}$ period fast magnetosonic foreshock waves: A Cluster case study, Geophys. Res. Lett., 31, L04804, doi:10.1029/2003GL018897, 2004.

Eastwood, J., Lucek, E. A., Mazelle, C., Meziane, K., Narita, Y., Pickett, J., and Treumann, R.: The Foreshock, Space Sci. Rev., 118, 41-94, 2005.

Eriksson S., Øieroset, M., Baker, D. N., Mouikis, C., Vaivads, A., Dunlop, M. W., Rème, H., Ergun, R. E., Balogh, A.: Walén and slow-mode shock analyses in the near-Earth magnetotail in connection with a substorm onset on 27 August 2001, J. Geophys. Res., 109, A10212, doi:10.1029/2004JA010534, 2004.

Fitzenreiter, R. J., Klimas, A. J., and Scudder, J. D.: Detection of bump-on-tail reduced electron velocity distributions at the electron foreshock boundary, Geophys. Res. Lett., 11, 496-499,
1984.

Fu, H. S., Cao, J. B., Zhang, T. L., Rème, H., and Lucek, E.: Statistical study of the solar wind deceleration in the Earth's foreshock region, Chinese J. Geophys., 52(4), 895-901, 2009.

Gosling, J. T., Thomsen, M. F., Bame, S. J., Feldman, W. C., Paschmann, G., and Sckopke, N.: Evidence for specularly reflected ions upstream from the quasi-parallel bow shock, Geophys.Res. Lett., 9, 1333-1336, 1982.

Hones Jr., E. W.: Substorm processes in the magnetotail: Comments on "On hot tenuous plasma, fireballs, and boundary layers in the Earth's magnetotail” by L. A. Frank et al., J. Geophys. Res., 82, 5633-5640, 1977.

Hones, E. W.: Transient phenomena in the magnetotail and their relation to substorms, Space Sci. Rev., 23, 393-410, 1979.

Hoshino, M., Mukai, T., Shinohara, I., Saito, Y., and Kokubun, S.: Slow shock downstream structure in the magnetotail, J. Geophys. Res., 105, 337-347, 2000.

Imada, S., Nakamura, R., Daly, P. W., Hoshino, M., Baumjohann, W., Muhlbachler, S., Balogh, A., and Rème, H.: Energetic electron acceleration in the downstream reconnection outflow region, J. Geophys. Res., 112, A03202, doi:10.1029/2006JA011847, 2007.

Johnstone, A. D., Alsop, C., Burge, S., Carter, P. J., Coates, A. J., Coker, A. J., Fazakerley, A. N., Grande, M., Gowen, R. A., Gurgiolo, C., Hancock, B. K., Narheim, B., Preece, A., Sheather, P. H., Winningham, J. D., and Woodliffe, R. D.: PEACE: A Plasma Electron and Current Experiment, Space Sci. Rev., 79, 351-398, 1997.

Le, G. and Russell, C. T.: A study of ULF wave foreshock morphology - I: ULF foreshock boundary, Planet. Space Sci., 40(9), 1203-1213, 1992.

Lembège, B. and Savoini, P.: Formation of Reflected Electrons Bursts by the Nonstationarity and Nonuniformity of a Collisionless Shock Front, J. Geophys. Res., 107, 1037, doi:10.1029/2001JA900128, 2002.

Lembège, B., Giacalone, J., Scholer, M., Hada, T., Hoshino, M., Krasnoselskikh, V., Kucharek, H., Savoini, P., and Terasawa, T.: Selected problems in collisionless-shock physics, Space Sci. Rev., 110, 161-226, 2004.

Meziane, K. and d'Uston, C.: A statistical study of the upstream intermediate ion boundary in the Earth's foreshock, Ann. Geophys., 16, 125-133, 1998, http://www.ann-geophys.net/16/125/1998/.

Meziane, K., Mazelle, C., Lin, R. P., Le Quéau, D., Larson, D. E., Parks, G. K., and Lepping, R. P.: Three-dimensional observations of gyrating ion distributions far upstream from the Earth's bow shock and their association with low-frequency waves, J. Geophys. Res., 106, 5731-5742, 2001.

Meziane, K., Wilber, M., Mazelle, C., Le Quéau, D., Kucharek, H., Lucek, E. A., Rème, H., Hamza, A. M., Sauvaud, J. A., Bosqued, J. M., Dandouras, I., Parks, G. K., McCarthy, M., Klecker, B., Korth, A., Bavassano-Cattaneo, M. B., and Lundin, R. N.: Simultaneous observations of field-aligned beams and gyrating ions in the terrestrial foreshock, J. Geophys. Res., 109, A05107, doi:10.1029/2003JA010374, 2004.

Parks, G. K., Lee, E., Mozer, F., Wilber, M., Lucek, E., Dandouras, I., Rème, H., Mazelle, C., Cao, J. B., Meziane, K., Goldstein, M. L., and Escoubet, P.: Larmor radius size density holes discovered in the solar wind upstream of Earth's bow shock, Phys. Plasmas, 
13, 050701, doi:10.1063/1.2201056, 2006.

Paschmann, G., Sckopke, N., Papamastorakis, I., Asbridge, J., Bame, S., and Gosling, J.: Energetization of solar wind ions by reflection from the Earth's bow shock, J. Geophys. Res., 85, 4689-4693, 1980.

Rème, H., Aoustin, C., Bosqued, J. M., Dandouras, I., et al.: First multispacecraft ion measurements in and near the Earth's magnetosphere with the identical Cluster ion spectrometry (CIS) experiment, Ann. Geophys., 19, 1303-1354, 2001, http://www.ann-geophys.net/19/1303/2001/.

Savoini, P. and Lembège, B.: Two-dimensional simulations of a curved shock: self-consistent formation of the electron foreshock, J. Geophys. Res., 106, 12975-12992, 2001.

Schwartz, S. J., Thomsen, M. F., and Gosling, J. T.: Ions upstream of the Earth's bow shock - A theoretical comparison of alternative source populations, J. Geophys. Res., 88, 2039-2047, 1983.

Sergeev, V., Runov, A., Baumjohann, W., Nakamura, R., Zhang, T. L., Volwerk, M., Balogh, A., Reme, H., Sauvaud, J. A., Andre, M., and Klecker, B.: Current sheet flapping motions and structure observed by Cluster, Geophys. Res. Lett., 30, 1327, doi:10.1029/2002GL016500, 2003.

Slavin, J. A., Smith, E. J., Tsurutani, B. T., Sibeck, D. G., Singer, H. J., Baker, D. N., Gosling, J. T., Hones, E. W., and Scarf, F. L.: Substorm associated traveling compression regions in the distant tail: ISEE-3 geotail observations, Geophys. Res. Lett., 11, 657660, 1984.
Slavin, J. A., Baker, D. N., Craven, J. D., Elphic, R. C., Fairfield, D. H., Frank, L. A., Galvin, A. B., Hughes, W. J., Manka, R. H., Mitchell, D. G., Richardson, I. G., Sanderson, T. R., Sibeck, D. J., Smith, E. J., and Zwickl, D.: CDAW-8 observations of plasmoid signatures in the geomagnetic tail: An assessment, J. Geophys. Res., 94, 15153-15175, 1989.

Slavin, J. A., Tanskanen, E. I., Hesse, M., Owen, C. J., Dunlop, M. W., Imber, S., Lucek, E. A., Balogh, A., and Glassmeier, K.-H.: Cluster observations of traveling compression regions in the near-tail, J. Geophys. Res., 110, A06207, doi:10.1029/2004JA010878, 2005.

Southwood, D. J. and Kivelson, M. G.: On the form of the flow in the magnetosheath, J. Geophys. Res., 97, 2873-2879, 1992.

Ugai, M.: Computer studies on dynamics of a large-scale magnetic loop by the spontaneous fast reconnection model, Phys. Plasmas, 3, 4172-4180, 1996.

Ugai, M.: Basic physical mechanism of reconnection development and magnetic loop dynamics, J. Geophys. Res., 104, 6929-6939, 1999. 